\title{
A First Step Towards Practical Single Cell Proteomics: A Microfluidic Antibody Capture Chip with TIRF Detection
}

\author{
Ali Salehi-Reyhani $*^{*} \dagger^{a}$, Joseph Kaplinsky $\dagger^{a}$, Edward Burgin $\uparrow^{a}$, Miroslava Novakova ${ }^{a}$, Andrew J. de Mello ${ }^{a b}$, \\ Richard H. Templer ${ }^{a b}$, Peter Parker ${ }^{d}$, Mark A. A. Neil ${ }^{a c}$, Oscar Ces ${ }^{a b}$, Paul French ${ }^{a c}$, Keith R. Willison ${ }^{a e}$ and \\ ${ }_{5}$ David Klug*ab
}

We have developed a generic platform to undertake the analysis of protein copy number from single cells. The approach described here is 'all-optical' whereby single cells are manipulated into separate analysis chambers using an optical trap; single cells are lysed by a shock wave caused by laser-induced microcavitation; and the protein released from a single cell is measured by total 10 internal reflection microscopy as it is bound to micro-printed antibody spots within the device. The platform was tested using GFP transfected cells and the relative precision of the measurement method was determined to be $88 \%$. Single cell measurements were also made on a breast cancer cell line to measure the relative levels of unlabelled human tumour suppressor protein p53 using a chip incorporating an antibody sandwich assay format. These results suggest that this is a viable 15 method for measuring relative protein levels in single cells.

\section{Introduction}

There are a number of reasons to analyse protein copy number and protein post translational modifications at the level of single cells. Firstly, there is the case of rare or precious cells 20 where the limited quantities available give the researcher no choice. A good example of this is the analysis of circulating tumour cells where there can be as few as one cell in a clinical blood sample ${ }^{1}$. Secondly is the situation where cell to cell variation masks the properties of interest or where the 25 variation from cell to cell actually is the property of interest. Examples of this can be found in the study of genetically identical bacterial populations ${ }^{2}$. Finally, there is the interest in sub-cellular properties such as location of cellular components and/or co-location of cellular components. Despite recent 30 advances that offer control of single cells ${ }^{3}$, in terms of manipulation and sorting and the ability to measure gene expression, the need to measure protein copy number remains unmet. Measuring protein copy number in single cells and related quantities such as levels of phosphorylation and 35 protein-protein interaction is the basis of single cell proteomics.

The combination of fluorescent labelling of proteins, particularly using genetically engineered constructs, such as fluorescent fusion proteins, and modern microscopy has 40 proven to be a particularly powerful combination for single cell analysis. This approach has in recent years started to uncover the connectivity of protein networks including some fascinating reports of pulsitile behaviour such as in the case of the creation of pulses of p53 in response to a DNA-damaging 45 stimulus $^{4}$. The combination of microscopy and fusion protein labelling is extremely powerful and will no doubt continue to provide important new insights. It does, however, have some significant limitations. Firstly, there is always the concern that the inclusion of fusion proteins can alter the biology
50 observed $^{5,}{ }^{6}$. Perhaps more limiting however are the restrictions caused by the need to use genetically engineerable cells. This is particularly problematic for human samples and in particular for human clinical samples. Moreover, although immortal cell lines are easily engineered and studied, many of 55 these cell lines have biology which is greatly altered from the biology of the human tissue from which it originates. This is due to a combination of their origins as cancerous cells and their genetic instability over many years of culture in many different laboratories around the world under many different ${ }_{60}$ conditions $^{7}$. Furthermore, there is no practicable method for engineering human clinical samples either from healthy volunteers or from sick patients such that they can be studied using fusion protein labels. Finally, there is the restriction on multiplexing signals from multiple labels for multiple ${ }_{65}$ proteins. The current practical limit for live cells is three or four different labels, and even then, if stable cell lines are required, the practicalities mean that such experiments are rarely undertaken.

In this paper we outline a single cell proteomic technology 70 that is relatively scalable in terms of the ability to study multiple proteins in parallel. More importantly, it requires no genetic engineering of the samples and can therefore be applied to human tissue and clinical samples. It can also in principle be adapted to the study of protein-protein 75 interactions and phosophorylation.

The single cell proteomic technology described here includes a number of components that are combined within a single experimental arrangement to produce a practicable workflow. The elements of this process are 1) single cell collection, 80 manipulation and reliable loading of cells into the analysis device; 2) reproducible lysis of the cells and 3) analysis of the contents of the lysed cell. Each of these steps has many independent solutions and the overall workflow can therefore 

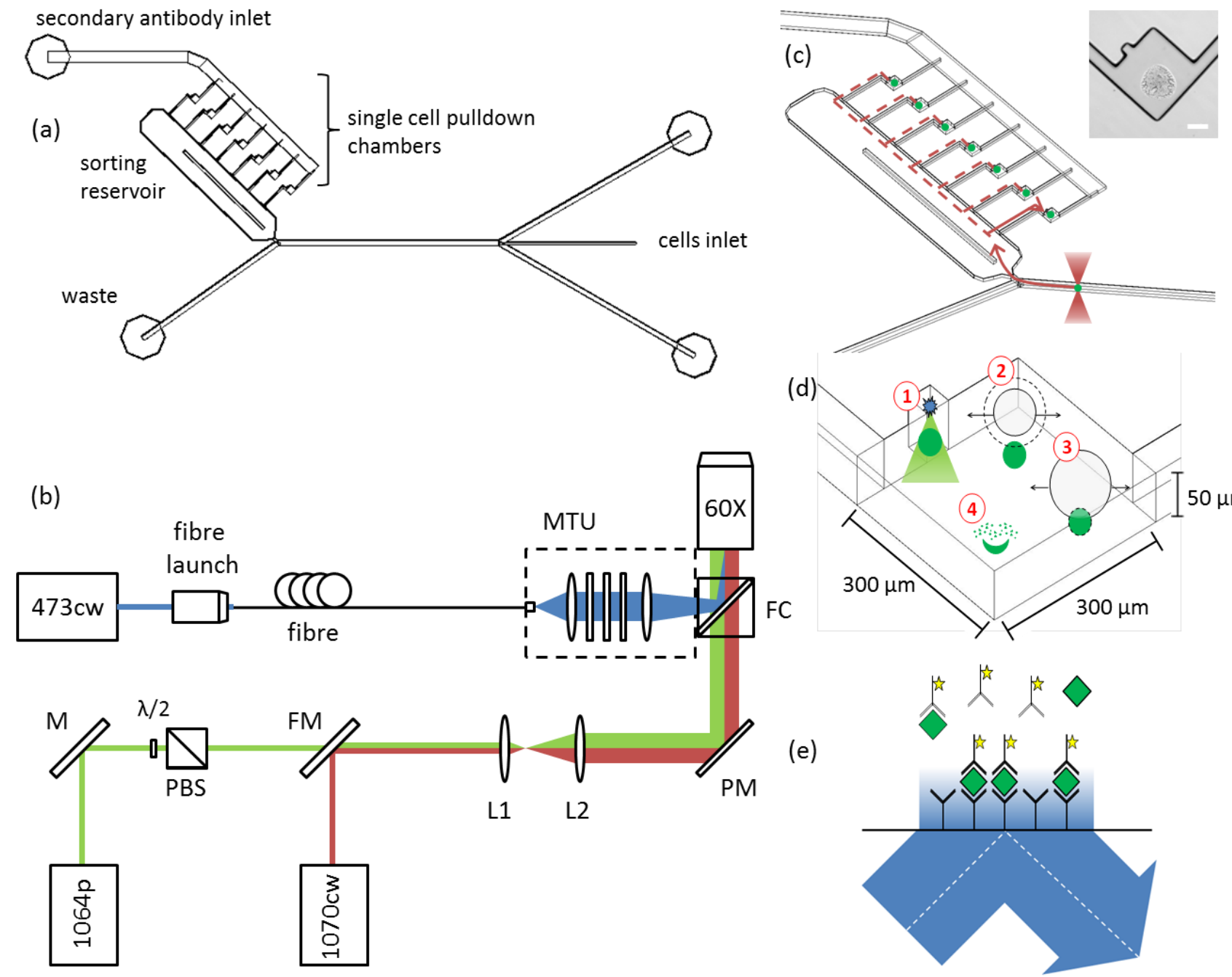

(d)

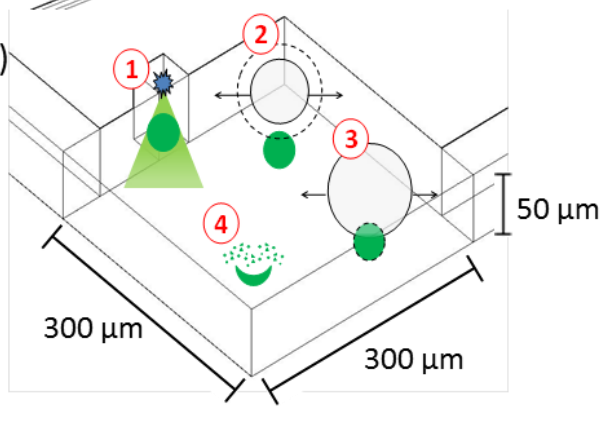

(e)

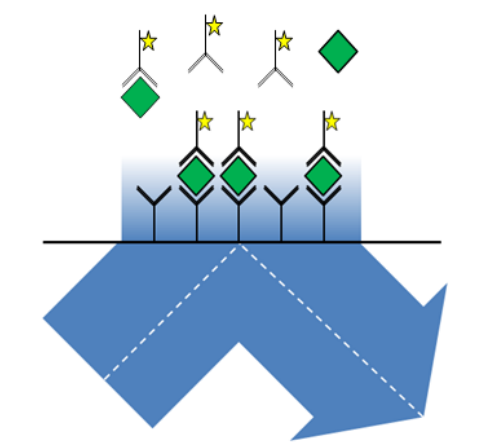

Fig. 1 (a) Schematic of the device used for single cell experiments. (b) Schematic of the optical setup. $473 \mathrm{cw}=\mathrm{cw}$ TIRF laser at $\lambda=473 \mathrm{~nm}, 1064 \mathrm{p}=$ pulsed Nd:YAG laser at $\lambda=1064 \mathrm{~nm}, 1070 \mathrm{cw}=\mathrm{cW}$ Ytterbium fibre laser at $\lambda=1070 \mathrm{~nm}, \mathrm{M}=$ mirror, $\mathrm{FM}=$ flip mirror, PM $=$ periscope mirrors, $\lambda / 2=$ halfwave plate, PBS = polarising beam splitter, L1 \& L2 =lenses in beam expander , MTU = Nikon motorised TIRF unit, FC = filter cube. (c) - (e) 3-step 5 workflow employing optical methods to isolate and trap cells, lyse and subsequently analyse protein content in a single cell. (c) The optical trap is used to move cells (green circles) from flow to analysis chambers. Inset: brightfield image of an antibody spot aligned within a chamber. Scale bar $=100 \mu \mathrm{m}$. (d) Single cells (green circles) are lysed by the delivery of a single $6 \mathrm{~ns}$ pulse at $\lambda=1064 \mathrm{~nm} 10 \mu \mathrm{m}$ above the centre of the cell. (1) At sufficient irradiance the medium breaks down to form a localised plasma; (2) An outwardly propagating shockwave and an expanding cavitation bubble are produced; (3) the cell is lysed due to shear stress from the expanding cavitation bubble; and (4) cellular constituents are released into the chamber. (e) 10 Single cell protein levels are measured using an antibody spot. Chamber volume is $4.6 \mathrm{~nL}$ and results in favourable kinetics. By employing TIRF, only fluorophores within $200 \mathrm{~nm}$ of the surface are imaged, which are assumed to be antibody/antigen bound.

be constituted in a variety of ways. In this paper we give the first example of such a workflow exemplified by three particular choices of the elements described above. Crucially, 15 these elements allow the study in principle of any protein of interest, in any cell type of interest, with single cell sensitivities and without the need for genetic engineering. We name this class of device Microfluidic Antibody Capture chips, or MAC chips for short. We show that the precision 20 and fidelity of the combined workflow is sufficient to produce data that will is revealing of cell to cell variation in protein copy number and demonstrate the first measurement of 'native' cell to cell protein copy number variation using the tumour suppressor protein p53 as an example.

\section{Cell Culture and Preparation}

BE colon carcinoma cell line was used as previously described $^{8}$ and transfected to express cytoplasmic GFP. The cells were cultured using Dulbecco's Modified Eagles 30 Medium (DMEM; Invitrogen, UK)) supplemented with 10\% (v/v) foetal bovine serum (FBS; Sigma, UK). Cells that were used in experiments were detached from culture flasks using trypsin (Invitrogen, UK), which was inactivated by suspension in $4 \%$ bovine serum albumin (BSA; Sigma, UK) and 10\% ${ }_{35}$ culture medium in phosphate-buffered saline (PBS; Invitrogen, UK). A suspension of single cells was created by gentle agitation with a pipette before sieving through a nylon mesh of $40 \mu \mathrm{m}$ pitch (BD Falcon, UK) to remove any cell clumps. MDA-MB468 is a breast cancer cell line. The cells

\section{${ }_{25}$ Materials and Methods}


were cultured in a 1:1 mixture of DMEM and Ham's F12 nutrient mixture (DMEM/F12 HAM; Invitrogen, UK) media supplemented with $10 \%(\mathrm{v} / \mathrm{v})$ foetal bovine serum (FBS; Sigma, UK). Cells were detached from from culture flasks 5 using trypsin, which was inactivated by culture medium.

\section{Antibodies}

AntiGFP monoclonal antibody was raised in BALB/c mice and donated by Pr. P. Parker (Cancer Research UK, Lincoln's Inn Fields Laboratories). It was dialysed into phosphate 10 buffered saline (Invitrogen, UK), aliquoted and stored at -80 ${ }^{\circ} \mathrm{C}$ before use. P53 protein is detected using an antibody sandwich assay. The antip53 primary antibody is taken from a commercial test kit (p53/Mdm2; Enzo Life Science, UK) whose epitope is at the C-terminus of the protein. The 15 secondary antibody is the DO1 monoclonal antibody recognising amino acids 21-25 at p53's N-terminus and is fluorescently labelled with Alexa-Fluor 488 (sc-126; Santa Cruz, USA). All antibodies have been validated with native and non-native Western blots using cell lysate (data not 20 shown).

\section{Printing of Antibody Arrays}

Coverslips were surface derivitised with (3glycidoxypropyl)trimethoxy silane (GPTS; Sigma, UK). Slides were first cleaned by $30 \mathrm{~min}$ Sonication in ethanol 25 (VWR, UK), then immersed in $10 \%$ sodium hydroxide (VWR, $\mathrm{UK}$ ) for $1 \mathrm{~h}$. They were rinsed four times in water and twice in ethanol, then immersed in $2.5 \%$ GPTS in ethanol for $1 \mathrm{~h}$, followed by $10 \mathrm{~min}$ sonication to remove unreacted linker. Coverslips were forced-air dried and used immediately for 30 antibody printing. All antibodies were printed by an OmniGrid Micro microarrayer (Digilab, UK) using 946MP2 pins (ArrayIt, USA) and spotting solution comprised of commercial protein print buffer (ArrayIt, USA) supplemented with $0.5 \%$ PVA (molecular weight 9,000-10,000; Sigma, UK).

35 The final concentration of antiGFP and antip53 in spotting solution were $90 \mu \mathrm{g} / \mathrm{mL}$ and $62.5 \mu \mathrm{g} / \mathrm{mL}$, respectively. Spot to spot variation was determined by incubating antibody spots with purified antigen protein and $50 \%$ of print runs show a $7 \%$ spot to spot variation or lower; those print runs that do not 40 achieve this target are rejected.

\section{Microfluidic Device Fabrication}

The single cell analysis device (fig. 1a) was fabricated using SU-8-based soft-lithography techniques for PDMS ${ }^{9}$. PDMS was mixed at a ratio of 10:1 precursor to curing agent and 45 poured over the SU-8 mold before being degassed in a dessicator chamber and set to cure at $60^{\circ} \mathrm{C}$ for $2+$ hours. The $\mathrm{Si}$-wafer surface was passivated by vapour-deposition of (tridecafluoro-1,1,2,2-tetrahydrooctyl) trichlorosilane (SigmaAldrich, UK) under vacuum to enable easy lift-off of cured ${ }_{50}$ PDMS. The cured PDMS was removed from the mould and access holes were punched with a needle. The PDMS was bonded to a glass slide $(75 \mathrm{~mm} \times 50 \mathrm{~mm} \times 1 \mathrm{~mm})$ channel-side away from the glass surface after exposure to an air plasma for 1 minute (Harrick Plasma, USA). The microchannels are 55 sealed by a coverslip (thickness \#1.5; Corning, UK) upon which antibody patches are printed in defined locations using the Axsys software (Digilab, UK) to drive the microarrayer. The antibody microspots are aligned using a home built translation stage (inset, fig. 1c). Plasma treatment would 60 obviously destroy antibodies so only the PDMS surface is plasma treated for irreversible bonding; however, contact bonding alone is sufficient to maintain device integrity.

\section{Experimental system}

Cell manipulation, lysis and subsequent analysis are all ${ }_{65}$ performed using optical based methods. The beam paths are shown schematically in fig. $1 \mathrm{~b}$ and are all delivered via a single $60 \mathrm{x}$ NA=1.49 oil-immersion objective. The design of the optical trap is typical of setups described elsewhere ${ }^{10}$ and is formed of a single-beam produced by an Ytterbium fibre 70 laser (YLM-5; IPG Photonics, UK) at $\lambda=1070 \mathrm{~nm}$, with the objective focal and trapping planes being coincident. The objective lens back aperture is overfilled to ensure stable trapping at low powers, typically $50-75 \mathrm{~mW}$, measured at the back aperture. A trapped cell is effectively moved within 75 the microfluidic by translating a motorised stage.

Optical lysis is performed by the delivery of a single pulse of 6ns duration at $\lambda=1064 \mathrm{~nm}$ from a Q-sw Nd:YAG laser (Surelite SL I-10, Continuum, USA) and has been previously shown to preserve cellular protein ${ }^{11,12}$. Briefly, at sufficiently 80 high irradiance, non-linear light absorption in the medium causes it to breakdown resulting in the formation of localised plasma, which results in a sequence of shockwave propagation and cavitation bubble expansion and subsequent collapse ${ }^{13}$. The primary agent of cell lysis has been shown to be shear 85 stresses caused from the expansion of the cavitation bubble. The process is described in detail elsewhere ${ }^{14}$. Pulses, of energy defined by a half-wave plate and a polarisation sensitive beam splitter, are delivered $10 \mu \mathrm{m}$ above the centre of the cell to be lysed. The threshold for plasma formation is 90 defined as the pulse energy that results in a $50 \%$ probability of plasma formation. It was measured in $4 \%$ BSA in PBS by observing the incidence of bright flashes recorded by the camera for 50 pulses at varying energies and determined to be $9.9 \pm 0.4 \mu \mathrm{J}$ by fitting to a Gaussian error function ${ }^{15}$. For 95 single cell lysis pulse energy is set to $14.1 \pm 0.3 \mu \mathrm{J}$, which correspond to $100 \%$ probability of plasma formation and ensures cells will be lysed by delivery of a single pulse.

To measure primary antibody bound fluorescent analyte (GFP), or bound analyte/secondary antibody complex (p53), 100 objective-based total internal reflection microscopy is employed with an electron-multiplied CCD (IXON DU-897E; Andor Technologies, Ireland) for background discrimination and single molecule detection capability. A solid state $\mathrm{cw}$ laser (MBL-473-200; Laser 2000, UK) at $\lambda=473 \mathrm{~nm}$ is 105 coupled into a single-mode optical fibre which is connected to a motorised TIRF illumination unit (Nikon, Japan). The power at the back aperture is adjusted by neutral density filters to $1.5 \mathrm{~mW}$.

Laser light for optical trapping and lysis were introduced 110 through a back-port on the microscope and reflected to the sample by a single-edge dichroic (LPD01-532R-25; Laser 2000, UK). Laser lysis pulses are filtered by a long pass filter 
(a)

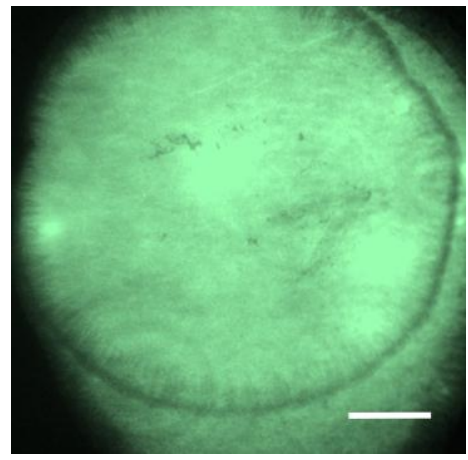

(b)

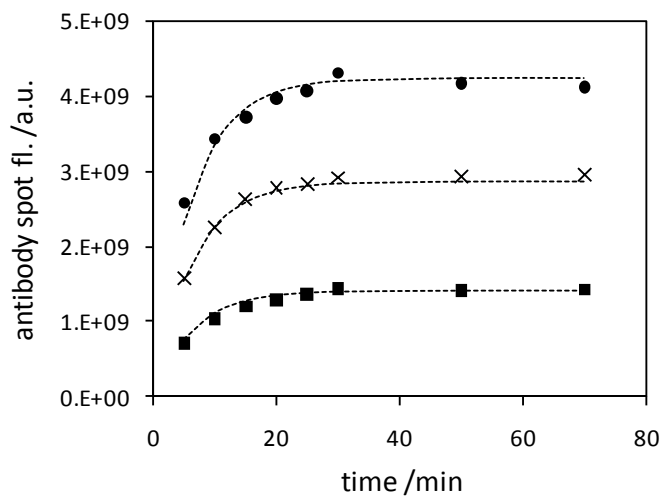

(c)

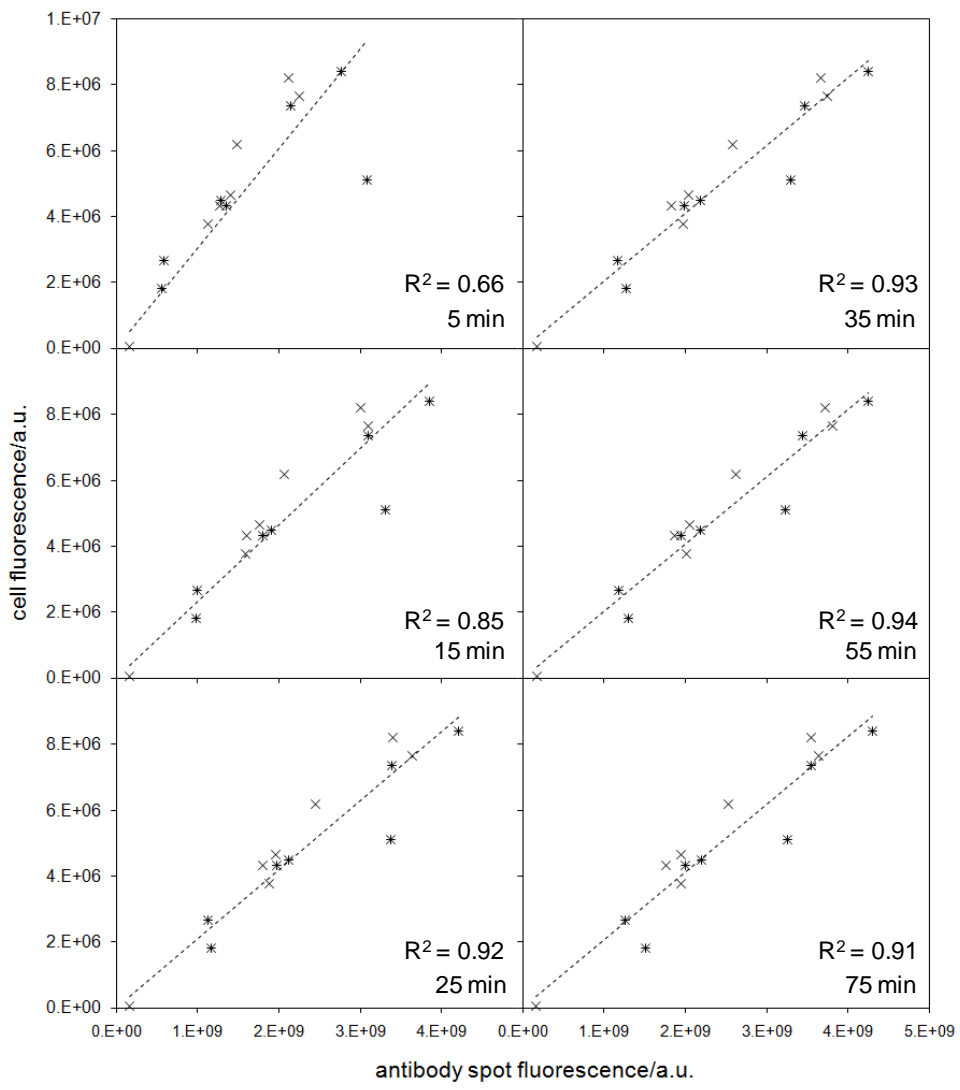

Fig. 2 (a) Typical antiGFP antibody spot imaged by TIRF. Scale bar $25 \mu \mathrm{m}$. The antibody spot is located in the lysis chamber, see figure $1 \mathrm{c}$ inset brightfield image of lysis chamber. (b) Kinetics of protein binding from 3 single BE cells whereby cytosolic GFP is freed upon lysis to bind to an antiGFP antibody spot within the same $4.6 \mathrm{~nL}$ chamber. The symbols, squares ( $\mathbf{\square})$, crosses $(X)$ and circles $(\bullet)$ represent 3 separate measurements of protein from a single cell binding to an antibody spot. The variation between the curves reflects the varying levels of GFP from cell to cell. (c) Correlation between the

5 amount of GFP in cells, as measured by widefield fluorescence, and the amount of GFP bound to each antibody spot, as measured by TIRF, over the time-course of the experiment. Widefield fluorescence levels are on the vertical axis and fluorescence from TIRF measurements of protein bound to the antibodies on the chip surface is on the horizontal axis. The stars and crosses represent data from two different experiments to give a feel of the reproducibility of the methodology. The straight lines are fits to the data and the high degree of correlation shows that the TIRF measurements faithfully recover the level of protein as measured by widefield microscopy.

10 at launch (LP03-532RS-25; Laser 2000, UK). For TIRF illumination a filter set (z488bp; Chroma, USA) was used.

\section{Experimental procedure}

An inverted microscope (Nikon Ti-E, Nikon, Japan) was used as the experimental platform. Antibody spot position within 15 each chamber was recorded using an encoded XY stage (Nikon, Japan). The device is degassed and allowed to fill with $4 \%$ BSA in PBS in a dessicator chamber. BSA serves to block the antibody spots from non-specific binding and to prevent cells sticking to the glass while being transported by 20 the optical trap. The device is returned to the microscope and connected via appropriate tubing to a syringe pump. BE cells were flowed down the main channel of the device at $1 \mu \mathrm{L} \mathrm{min}$ ${ }^{1}$ and upon nearing the entrance to the sorting chamber flow is stopped and a cell is moved, by the optical trap, to each 25 analysis chamber and placed within the small cubicle (fig. 1c). MDA-MB468 cells are introduced directly into the sorting chamber (fig. 1a) and moved from there. The cubicle protects the cell from displacement upon filling the chambers with secondary antibody for experiments with p53. In the case of ${ }_{30}$ GFP experiments, cells are imaged prior to lysis using widefield epi-fluorescence by excitation from a mercury lamp through a Nikon standard FITC filter set. Upon lysis of cells, antibody spots are imaged automatically in series by TIRF with the focal plane being maintained by a Nikon Perfect ${ }_{35}$ Focus System (Nikon, Japan).

\section{Single molecule counting}

Single molecule counting. The number of proteins captured per cell to the antibody spot can be estimated from dividing the image intensity by the known average intensity of a single 40 fluorescence reporter i.e the GFP protein or labelled secondary antibody. Images of 10 reporter molecules bound to an antibody spot are fit to a two-dimensional symmetrical Gaussian function. The parameters from all fits were averaged and used to estimate the number of proteins bound to 45 each antibody spot. The background transient count rate in one image frame is 100 proteins due to transient non-specific binding of the secondary antibodies so that in each frame 
$\sim 100$ of the counts are due to this background. Therefore, in our setup the limit of detection for antip53 is 100 proteins

\section{Results}

Before using MAC chips to study biology it is important to 5 demonstrate that they do indeed faithfully measure the protein level within a single cell and to establish the precision with which the MAC chips are able to do this. This is best done by measuring the correlation between the levels of protein measured by some other method with that determined using 10 the MAC chips themselves. A useful approach is to use conventional widefield fluorescence microscopy to measure the amount of fluorescently tagged protein within a single cell, and to correlate this against the level of protein measured uising the laser lysis, antibody capture and protein counting 15 methods of the workflow described. To do this we use a cell line stably transfected with GFP. GFP expression levels were measured for each cell by widefield fluorescence immediately prior to lysis and by TIRF subsequent to lysis and capture of the protein by the antibody patches. A time series of images

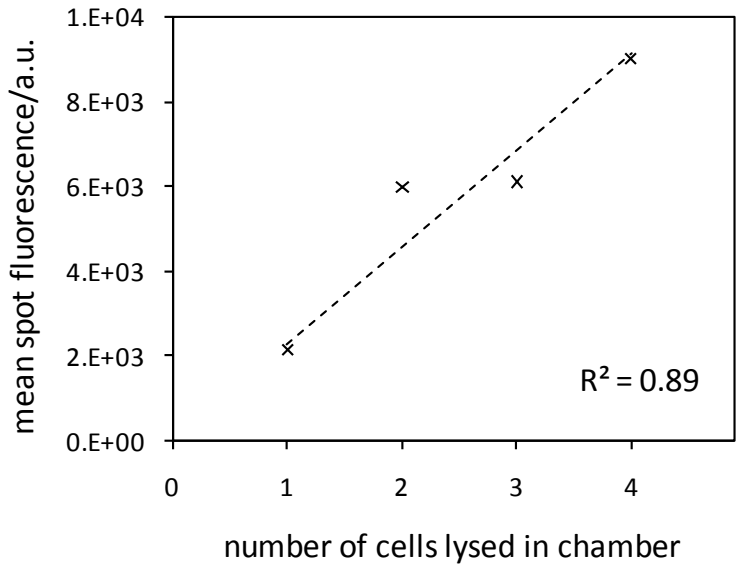

Fig. 3 One, two, three and four MDA-MB468 cells are loaded into separate chambers followed by lysis, capture of the protein and analysis. The figure shows that the p53 signal increases linearly with cell number and that the response is not saturating.

25 was acquired every $5 \mathrm{~min}$ for $30 \mathrm{~min}$ and every $20 \mathrm{~min}$ thereafter. A typical antiGFP antibody patch bound with GFP from a single cell is shown in fig. $2 \mathrm{a}$ and the time dependent accumulation of protein on the patches shown for measurement of 3 separate cells in fig. $2 \mathrm{~b}$. The binding 30 kinetics are approximately described by $\mathrm{B}(\mathrm{t})=\mathrm{T}-\mathrm{U}(\mathrm{t})=\mathrm{T}(1-$ $\mathrm{e}^{\wedge}-\mathrm{kt}$ ) where $\mathrm{T}, \mathrm{B}$ and $\mathrm{U}$ are the total, bound and unbound amounts of protein; $\mathrm{k}$ is the antibody binding constant, here $2.58 \times 10^{-3} \mathrm{~s}^{-1}$, and $\mathrm{t}$ is time. To minimise photobleaching, low excitation power and a short duty cycle of $0.075-0.3 \%$ 35 are employed; however photobleaching effects are effectively cancelled out when making relative measurements as we do in this paper so long as the experimental conditions are always the same. Simple calculations of the diffusion behaviour in these chambers suggest that the $95 \%$ of GFP binding should 40 be largely complete in approximately 20 minutes, which is broadly what we find in the data of fig. $2 \mathrm{~b}$ that shows a $1 / \mathrm{e}$ time constant of about 6.5 minutes.
Plots of cellular GFP fluorescence prior to lysis against antibody spot fluorescence post lysis is shown in fig. $2 \mathrm{c}$ for 45 six sequential time points. The number of GFPs captured per cell can be estimated by single molecule counting. The number of GFPs bound to each spot at steady state was determined. The minimum number of GFPs bound to a spot was $0.41 \times 10^{5}$ GFPs whereas the maximum was $2.7 \times 10^{5}$ ${ }_{50}$ GFPs. At this point we cannot say definitively whether each measurment accurately reflects the absolute number of GFP molecules from each cell as we do not currently know for certain what the capture efficiency of these particular devices with this particular antibody is.

${ }_{55}$ The precision of the MAC chips in measuring the relative levels of protein can be given a lower limit by making the assumption that the widefield fluorescence measurements are perfectly precise. In this case the standard deviation between the protein levels measured by fluorescence and those from ${ }_{60}$ the MAC chips is found to be $88 \%$. This means that the precision of our method is $88 \%$ at worst and could in fact be better than this depending on the actual precision of the widefield fluorescence measurement methodology.

In order to make measurements on unlabelled proteins and to 65 demonstrate the generality of the methodology we have also employed a sandwich assay format where a primary antibody printed onto the glass surface captures the protein of interest while a secondary antibody carrying a fluorescent label, present in the chambers at $50 \mathrm{ng} / \mathrm{mL}$, also binds to the 70 captured protein in order to make each protein detectable by TIRF. To demonstrate this capability we chose to measure the amount of p53 in the breast-cancer cell line MDA-MB468. Using single molecule counting, it is estimated that $1500 \pm$ 200 proteins are captured on average from each cell. In order 75 to demonstrate the linearity of the flurorescent signal with protein copy number, 1-4 cells were deposited in chambers and lysed (fig. 3). As can be seen, the fluorescence increases linearly with the number of cells lysed. Those deviations from linearity that are visible from the straight line fit to the data 80 almost certainly reflect genuine variation in protein copy number from cell to cell.

$\mathrm{Hu}$ et al., using an ELISA kit, measured p53 protein levels in a cell line where p53 expression was regulated by a tetracycline promoter ${ }^{16}$. Minimum levels were measured at $850.57 \times 10^{5}$ which increased to $5.9 \times 10^{5}$ upon complete withdrawal of tetracycline. Ma et al. have made similar measurements ${ }^{17}$ in 7 different cell lines and report p53 levels from $2-20 \times 10^{4}$ per cell, 5 lines of which with levels less than $5 \times 10^{4}$. It is highly likely that each cell contains 90 significantly more p53 than the 1500 copies captured by our chips. The relatively low number that we detect almost certainly reflects the high affinity of p53 for DNA and for other proteins, the binding of which is likely to obscure the recognition epitope for either the primary or secondary 95 antibodies. For now therefore, and in the absence of any further information, the 1500 copies captured are probably representative of the fraction of p53 unbound to DNA or other proteins. Nevertheless it proves the principle that we can reliably determine relative levels of protein in single cells 100 without the need to resort to labelling of the proteins. 


\section{Discussion}

One of the more important applications of this technology is likely to be the study of protein-protein interactions using a primary antibody against one protein and a secondary against ${ }_{5}$ its binding partner. In these cases it is important that the epitopes recognised by the antibodies differ from those recognised by the proteins. In the experiments reported here we are in effect measuring a popluation of p53 unbound to DNA or other proteins since the secondary epitope (aa 21-25) 10 overlaps the Mdm2 binding pocket (aa 18-26) and this may prevent recognition by the secondary ${ }^{18}$ antibody and therefore prevent detection.

The current design and fabrication methods used in our chips leads to a $50 \%$ failure rate, largely due to the strict 15 requirement upon antibody spot quality for single cell measurements. The demonstration devices used in this proof of principle study have only 7 chambers but this could be increased as long as the cells can be loaded in an effective and efficient way. The optical trap may not be ideal for trapping 20 large numbers of cells. However, for studies of rare cells such as circulating tumour cells (CTCs), where there are often only 1-10 CTCs per patient sample, the use of optical trapping can be a particularly good solution.

\section{Conclusions}

${ }_{25}$ We have demonstrated the ability count protein copy number from single cells in a manner which could be applied in principle to any set of proteins and for any cell type without the need for genetic engineering. The precision of the measurements is shown to be $\sim 88 \%$, easily sufficient for 30 studying the variation in protein copy number from cell to cell.

\section{Acknowledgements}

We thank Heather Rada for excellent support in cell culture and antibody qualification. This work was supported by the ${ }_{35}$ Single Cell Proteomics Project of the Institute of Chemical Biology and funded by the UK Engineering and Physical Sciences Research Council (EP/C54269X/1 and EP/G00465X/1).

\section{Notes and references}

$4{ }^{a}$ Single Cell Proteomics Project, Institute of Chemical Biology, Imperial College London, Exhibition Road, London, SW7 2AZ, UK; E-mail: ali.salehi-reyhani@imperial.ac.uk,d.klug@imperial.ac.uk

${ }^{b}$ Department of Chemistry, Imperial College London, Exhibition Road, London, SW7 2AZ, UK

$45{ }^{c}$ Optics \& Photonics Group. Department of Physics, Imperial College London, Exhibition Road, London, SW7 2AZ, UK

${ }^{d}$ Cancer Research UK, Lincoln's Inn Fields, London, WC2A 3PX, UK

${ }^{e}$ The Institute of Cancer Research, Chester Beatty Laboratories, 237

Fulham Road, London, SW3 6JB, UK

$50 \dagger$ Authors contributed equally to this work.

1. A. G. J. Tibbe, M. C. Miller and L. W. M. M. Terstappen, Cytom Part A, 2007, 71A, 154-162.

55 2. A. Raj and A. van Oudenaarden, Cell, 2008, 135, 216-226.
3. A. Schmid, H. Kortmann, P. S. Dittrich and L. M. Blank, Current Opinion in Biotechnology, 2010, 21, 12-20.

4. E. Batchelor, C. S. Mock, I. Bhan, A. Loewer and G. Lahav, Molecular Cell, 2008, 30, 277-289.

60 5. D. A. Hanson and S. F. Ziegler, J Negat Results Biomed, 2004, 3, 13.

6. B. Badrian and M. A. Bogoyevitch, DNA Cell Biol, 2007, 26, 727736.

7. J. F. Millau, S. Mai, N. Bastien and R. Drouin, Bioessays, 2010, 32, 65 392-400.

8. P. M. P. Lanigan, T. Ninkovic, K. Chan, A. J. de Mello, K. R. Willison, D. R. Klug, R. H. Templer, M. A. A. Neil and O. Ces, Lab Chip, 2009, 9, 1096-1101.

9. Y. N. Xia and G. M. Whitesides, Annual Review of Materials $70 \quad$ Science, 1998, 28, 153-184.

10. A. Ashkin, J. M. Dziedzic, J. E. Bjorkholm and S. Chu, Opt Lett, 1986, 11, 288-290.

11. R. B. Brown and J. Audet, Cytom Part A, 2007, 71A, 882-888.

12. H. Li, C. E. Sims, M. Kaluzova, E. J. Stanbridge and N. L. Allbritton, 75 Biochemistry-Us, 2004, 43, 1599-1608.

13. K. R. Rau, A. Guerra, A. Vogel and V. Venugopalan, Appl Phys Lett, 2004, 84, 2940-2942.

14. K. R. Rau, P. A. Quinto-Su, A. N. Hellman and V. Venugopalan, Biophysical Journal, 2006, 91, 317-329.

80 15. A. Vogel, K. Nahen, D. Theisen and J. Noack, Ieee J Sel Top Quant, 1996, 2, 847-860.

16. W. W. Hu, Z. H. Feng, L. Ma, J. Wagner, J. J. Rice, G. Stolovitzky and A. J. Levine, Cancer Res, 2007, 67, 2757-2765.

17. L. Ma, J. Wagner, J. J. Rice, W. W. Hu, A. J. Levine and G. A. 85 Stolovitzky, P Natl Acad Sci USA, 2005, 102, 14266-14271.

18. S. W. Chi, S. H. Lee, D. H. Kim, M. J. Ahn, J. S. Kim, J. Y. Woo, T. Torizawa, M. Kainosho and K. H. Han, J Biol Chem, 2005, 280, 38795-38802. 